\title{
Scaling of structure and electrical properties in ultrathin epitaxial ferroelectric heterostructures
}

\author{
V. Nagarajan ${ }^{a)}$ \\ School of Materials Science and Engineering, University of New South Wales, Sydney NSW 2052, Australia \\ J. Junquera ${ }^{\text {b) }}$ \\ Departmento de Ciencias de la Tierra y Física de la Materia Condensada. Universidad de Cantabria, \\ Santander 39005, Spain \\ J. Q. He, C. L. Jia, and R. Waser \\ Centre of Nanoelectronic Systems for Information Technology, IFF, Forschungszentrum Juelich, \\ D52425 Juelich, Germany \\ K. Lee, Y. K. Kim, and S. Baik \\ Department of Materials Science and Engineering, Pohang University of Science and Technology, \\ Pohang 790-784, Korea \\ T. Zhao and R. Ramesh \\ Department of Materials Science, University of California, Berkeley, California 94720 and Department \\ of Physics, University of California, Berkeley, California 94720 \\ Ph. Ghosez \\ Département de Physique, Université de Liège, Bâtiment B-5, B-4000 Sart-Tilman, Belgium \\ K. M. Rabe \\ Department of Physics and Astronomy, Rutgers University, Piscataway, New Jersey 08854
}

(Received 16 August 2005; accepted 22 November 2005; published online 15 September 2006; publisher error corrected 22 September 2006)

\begin{abstract}
Scaling of the structural order parameter, polarization, and electrical properties was investigated in model ultrathin epitaxial $\mathrm{SrRuO}_{3} / \mathrm{PbZr}_{0.2} \mathrm{Ti}_{0.8} \mathrm{O}_{3} / \mathrm{SrRuO}_{3} / \mathrm{SrTiO}_{3}$ heterostructures. High-resolution transmission electron microscopy images revealed the interfaces to be sharp and fully coherent. Synchrotron $\mathrm{x}$-ray studies show that a high tetragonality $(c / a \sim 1.058)$ is maintained down to $50 \AA$ thick films, suggesting indirectly that ferroelectricity is fully preserved at such small thicknesses. However, measurement of the switchable polarization $(\Delta P)$ using a pulsed probe setup and the out-of-plane piezoelectric response $\left(d_{33}\right)$ revealed a systematic drop from $\sim 140 \mu \mathrm{C} / \mathrm{cm}^{2}$ and $60 \mathrm{pm} / \mathrm{V}$ for a $150 \AA$ thick film to $11 \mu \mathrm{C} / \mathrm{cm}^{2}$ and $7 \mathrm{pm} / \mathrm{V}$ for a $50 \AA$ thick film. This apparent contradiction between the structural measurements and the measured switchable polarization is explained by an increasing presence of a strong depolarization field, which creates a pinned $180^{\circ}$ polydomain state for the thinnest films. Existence of a polydomain state is demonstrated by piezoresponse force microscopy images of the ultrathin films. These results suggest that the limit for a ferroelectric memory device may be much larger than the fundamental limit for ferroelectricity.

(c) 2006 American Institute of Physics. [DOI: 10.1063/1.2337363]
\end{abstract}

\section{INTRODUCTION}

The reorientable electric polarization of ferroelectric materials has long been of interest for its potential in high density information storage. ${ }^{1,2}$ One of the most crucial questions regarding the densities and performance that can ultimately be achieved is that of the size effect ${ }^{3-5}$ on the magnitude and stability of uniform switchable polarization. In the past few years, careful measurements on high-quality ultrathin films ${ }^{6-14}$ and related theoretical models ${ }^{15-18}$ for thin films have shown that a ferroelectric instability can be exhibited by perovskite oxide films as thin as a few lattice constants. This has led to renewed interest in understanding the scaling of ferroelectric properties with thickness in the ultrathin re-

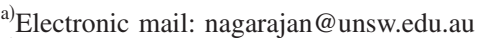

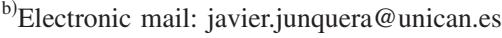

gime. To preserve states with uniform polarization, the compensation of the depolarization field has been identified as a crucial factor. ${ }^{18-21}$ On $\mathrm{SrTiO}_{3}$ (STO) insulating substrates, x-ray studies of films of $\mathrm{PbTiO}_{3}$ (PTO) exhibit a polar distortion, but to reduce depolarization energy the system forms periodic $180^{\circ}$ ferroelectric stripe domains. ${ }^{9,11}$ Metallic electrodes under short-circuit conditions should provide a mechanism for compensation of the depolarization field that acts to stabilize the uniformly polarized state. However, in real conductors the screening is imperfect. As the films get thinner, the residual field in the ferroelectric due to the surface-dipole density at the ferroelectric/electrode interface grows, ${ }^{18,20}$ resulting in renormalization of the coercive field, ${ }^{22}$ reduction in polarization of uniformly polarized films, ${ }^{23}$ and ultimately a possible destabilization of ferroelectricity in the thinnest films. 
For information storage technology, the figure of merit is the magnitude and stability of the switchable ferroelectric polarization. This is referred to as $\Delta P$ and ideally is twice the remnant polarization. Measurements of the thickness variation of the magnitude of $\Delta P$ for uniformly polarized films in the sub-100- $\AA$ thickness regime are therefore of particular interest, as this magnitude is expected to be modified by the residual depolarizing field and other corrections resulting from the imperfect screening in the electrodes. Despite its importance, and mainly due to the fact that polarization measurements for ultrathin films are particularly dominated by leakage, in a majority of experimental studies $\Delta P$ is not measured directly but inferred from the correlation with the measured lattice parameter, ${ }^{23}$ pyroelectric $^{6}$ or piezoelectric ${ }^{7}$ coefficients. This fact spoils the quantification of the switchable part of the net polarization.

Only very recently Kim et al. showed ferroelectric hysteresis loops for epitaxial $\mathrm{BaTiO}_{3}$ (BTO) films as thin as $50 \AA .{ }^{14}$ Structurally they observe that even the $50 \AA$ film exhibits $c$-axis value that is considerably larger than the bulk values for BTO while the polarization for the $50 \AA$ film is considerably reduced with respect to the bulk. They see a systematic decrease in the polarization as a function of reducing thickness, and their experimental data agree well with the theoretical first-principles based predictions of Junquera and Ghosez. ${ }^{18}$ In contrast a systematic study of PTO ultrathin epitaxial films by Lichtensteiger et al. showed a monotonic decrease in the lattice tetragonality with decreasing thickness. $^{23}$ Further for certain ferroelectrics a clear resistance switching effect was observed when scaled to very low thickness. $^{24,25}$ There is also theoretical understanding now that predicts tunneling effects in ultrathin ferroelectric barriers sandwiched between metal electrodes. ${ }^{26,27}$ Thus, there exists still a conundrum, particularly in epitaxial ultrathin heterostructures, as to how the lattice and polarization properties scale with thickness for different ferroelectric compositions.

To understand this we combined direct measurements of the switchable ferroelectric polarization and piezoelectric responses with information about the structure to study scaling effects in a model heterostructure system of high-quality ultrathin $\mathrm{PbZr}_{0.2} \mathrm{Ti}_{0.8} \mathrm{O}_{3}$ (PZT) films with $\mathrm{SrRuO}_{3}$ (SRO) electrodes. The thinnest films show a sharp drop in the measured $\Delta P$ and the out-of-plane piezoelectric constant but virtually no change in the lattice constants. This observation is explained by the depolarization field model, in which decreasing thickness leads to increases in the residual field that destabilize the system into a $180^{\circ}$ domain state similar to that observed on an insulating substrate. ${ }^{11,21}$

\section{EXPERIMENT}

Epitaxial PZT ultrathin films 50-500 $\AA$ thick were grown on SRO buffered (100) STO substrate by pulsed laser deposition. Details of the growth are given elsewhere. ${ }^{13}$ The maximum thickness of the PZT film presented in this study was limited to $500 \AA$ in order to maintain fully $c$-axis oriented films and avoid the formation of ferroelastic $a$-axis domains $\left(90^{\circ}\right.$ domains $)$. The microstructure and local distor- tion in ultrathin films were investigated by means of highresolution transmission electron microscopy (HRTEM). HRTEM images were obtained using a JEOL-4000EX highresolution electron microscope operating at $400 \mathrm{keV}$ with point to point resolution of $0.17 \mathrm{~nm}$.

Lattice constants for films greater than $500 \AA$ thickness were measured using a conventional $\mathrm{x}$-ray diffractometer fitted with thin film optics. The lattice parameters for the 50 and $80 \AA$ PZT films were investigated by synchrotron x-ray diffraction at the Pohang Light Source (PLS). The beam size at the focal point is typically less than $1 \mathrm{~mm}^{2}$. A scintillation detector was used to record the diffracted beam intensities.

To ascertain that the ultrathin films have low leakage paths, direct current atomic force microscopy (dc-AFM) was used to image leakage current paths of these ultrathin films. The images were obtained at room temperature and under $10^{-3}$ bar on a JEOL scanning tunneling microscope (STM)/ AFM with $1 \mathrm{pA}$ detection limit. This technique has been used to image dislocations in semiconductors, ${ }^{28}$ leakage in $\mathrm{SiO}_{2}$ metal-oxide-semiconductor (MOS) structures, ${ }^{29}$ nanoscale current transport, ${ }^{30}$ and screening phenomena in oxide ferroelectrics. ${ }^{31}$ In this technique a voltage is applied between a conducting tip and the sample and the resulting current is detected using a current preamplifier $/ I-V$ converter. The current images are obtained simultaneously with topography and hence allow the correlation of electrical properties with topological features.

Direct quantitative ferroelectric properties were evaluated using a pulse method using an atomic force microscope (AFM). ${ }^{32}$ This technique facilitates measurement of nanoscale submicron capacitors and with fast rise times, both very important to successfully test ferroelectric nanostructures. The pulsed probe method was chosen over the traditional $P-E$ hysteresis measurement as it is less convoluted by leakage and nonlinear dielectric effects. However, to check the existence of resistive switching effects, the thinnest film was swept under a voltage bias as would be for a hysteresis measurement. To avoid leakage paths submicron sized capacitors were created, especially for the thinnest film, using a modified lift-off process. The typical lateral area of the capacitors for the $50 \AA$ film was $0.125 \mu \mathrm{m}^{2}$, while the typical lateral areas for the thicker films were $4 \mu \mathrm{m}^{2}$. Quantitative piezoresponse force microscopy ${ }^{33}$ (PFM) was used to measure the piezoelectric $\left(d_{33}\right)$ responses. The deflection of the PFM tip was calibrated using an $X$-cut quartz crystal. Further details on the PFM calibration can be found in Ref. 34 .

\section{RESULTS AND DISCUSSION}

\section{A. Structural measurements}

Figure 1(a) is a cross-sectional HRTEM image of a PZT/ SRO/STO heterostructure (the presence of a top SRO hindered the resolution of the TEM image; for all electrical and piezoelectric measurements a top $\mathrm{SRO} / \mathrm{Pt}$ electrode was used), with the PZT of nominal thickness of $50 \AA$. In our previous report we presented cross-sectional HRTEM data on a film that was $40 \AA$ thick. ${ }^{13}$ In that case, the average lattice tetragonality ( $c / a$ ratio) was calculated based on the power spectrum of a lattice image to be 1.05 . In the present 

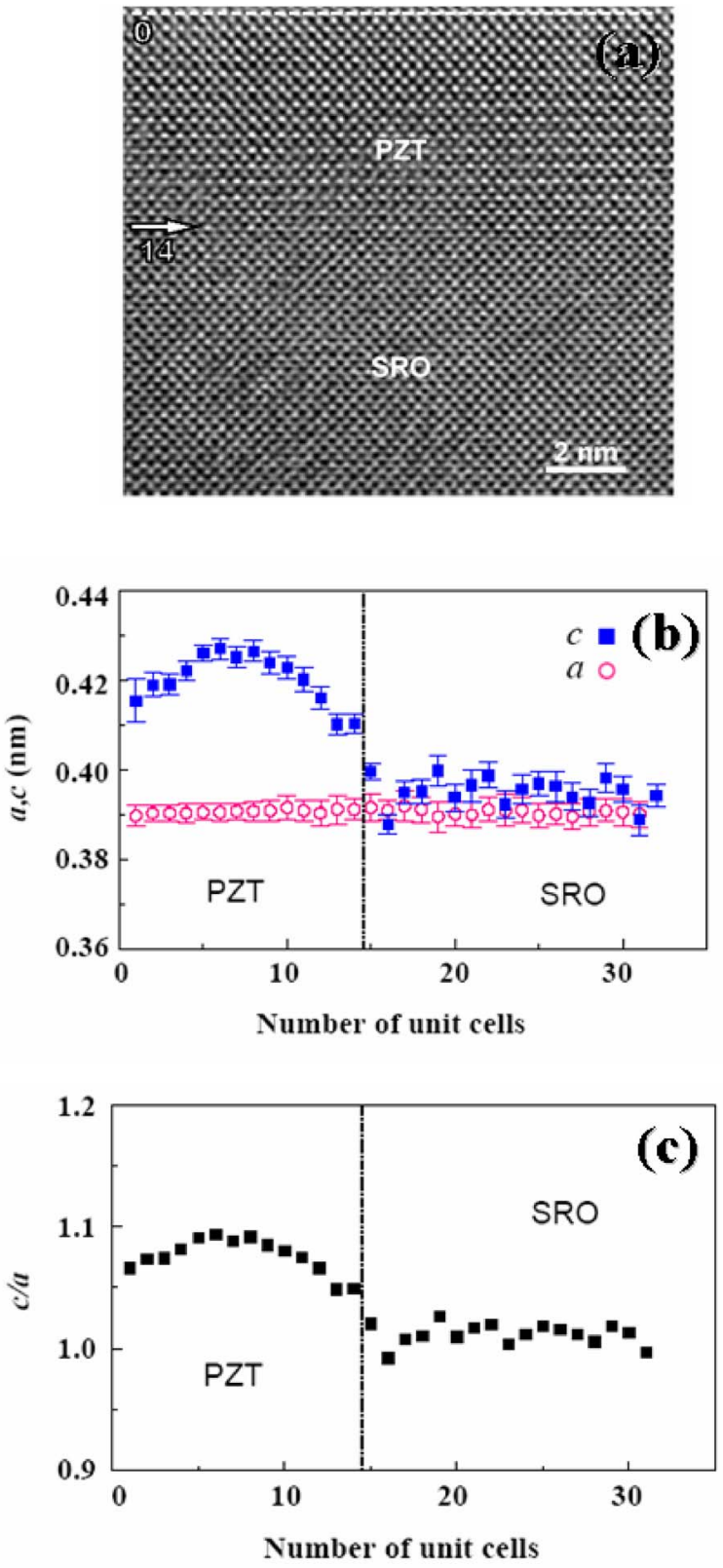

FIG. 1. (Color online) (a) High-resolution lattice image of the PZT film grown on SRO/STO. The film is 14 unit cell thick. The image shows a sharp and coherent interface between the ferroelectric and bottom electrode. (b) Out-of-plane, $c$, and in-plane, $a$, axis lattice parameter as a function of layer. The lattice parameter of $\mathrm{SrTiO}_{3}(3.905 \AA)$ was used to calibrate the values. (c) Local layer-by-layer lattice tetragonality.

report we show a detailed analysis of the local lattice strain of the film by lattice mapping directly on the image using a numerical center-of-mass approach. In the image of Fig. 1(a) the distance between the top free surface (dashed line) and the bottom ferroelectric-electrode interface (arrow) is approximately 14 layers, each a single unit cell thick. In Fig. 1(b) we plot the measured $c$ - and $a$-axis parameters, and in Fig. 1(c) we present the local layer-by-layer $c / a$ ratio. All parameters were calibrated with respect to the lattice parameter of the STO substrate, which was fixed at $3.905 \AA$. We find two key observations. Firstly, a slight decrease in the out-of-plane parameter, and hence in the tetragonality, is observed at the interfaces compared to the interior of the PZT layers; the exact reasons for this are unclear. Recently van Helvoort et al. used low-angle annular dark-field (ADF) scanning transmission electron microscopy to show that epitaxial $\mathrm{PbTiO}_{3} / \mathrm{SrTiO}_{3}(\mathrm{PTO} / \mathrm{STO})$ films display a strained interface. ${ }^{35}$ They too found that careful comparison between the lattice parameters in the volume of the thin films to the interface unveiled a reduced $c$-axis lattice parameter near the film/substrate interface. This trend may be compatible with the scenario of an extrapolated length ${ }^{15,17}$ for thin films, where in surface effects induce a suppression of the polarization and hence lattice parameter.

Secondly, the measured $c / a$ ratio (ranging from 1.07 to $\sim 1.09$ ) from the high-resolution image is higher than those reported by x-ray studies (as discussed in the next section) for this PZT composition. It is difficult to convolute if the high tetragonality is an intrinsic effect or due to, for example, thin film effects or residual strain. We point out that a tetragonality of 1.09 was obtained near domain walls in $\mathrm{PbTiO}_{3}$ thin films by direct analysis of HRTEM images, ${ }^{36}$ which is higher than the expected value of 1.06. There is no satisfactory quantitative explanation of this difference; thus the only inference from the HRTEM images is that the lattice is highly tetragonal.

Due to the above inherent issues with HRTEM analysis we further probed the structural parameters via $\mathrm{x}$-ray diffraction. Figure 2(a) is a $\theta-2 \theta$ scan of the $50 \AA$ sample using synchrotron source at PLS. The (001) PZT, (100) SRO, and (100) STO peaks are indicated on the graph. Again the lattice parameter of the STO substrate was used to calibrate the peak positions. From this graph we measure the out-of-plane lattice parameter to be $4.132 \AA$. To measure the in-plane scan the sample was tilted to obtain diffraction from the (102) reflection, with the results shown in Fig. 2(b). The in-plane lattice parameter was calculated to be $3.905 \AA$ and hence the tetragonality of the $50 \AA$ film is $\sim 1.058$. Similar scans were performed for each of the thickness in the series. Figure 2(c) is a graph of the tetragonality measured as a function of the film thickness. It demonstrates that for films less than $200 \AA$ thick there is an $\sim 1 \%$ increase in tetragonality compared to the bulk $c / a$ of 1.05 , indicating that the films are compressively stressed in plane; above this thickness the tetragonality relaxes by dislocation formation. Although there is some difference between the HRTEM and x-ray measurements, the overall conclusion is that a high tetragonality is maintained down to $50 \AA$ thickness. A similar trend was observed for BTO ultrathin films in the report by Kim et al. ${ }^{14}$ For films thinner than $350 \AA$ and down to $50 \AA$ a strong increase in the $c$ lattice parameter compared to bulk BTO (an elongation of 6\%) was observed, indicating a residual compressive strain in the plane of the substrate. For films thicker than $350 \AA$ the out-of-plane parameter relaxed to bulklike values-this was attributed to relaxation by misfit dislocations.

Due to the strong polarization-strain coupling ${ }^{37}$ in PTO rich systems a high tetragonality is associated with a high polarization. Thus the trend shown by the $c / a$ ratio as a function of thickness suggests that even below sub-100- $\AA$ thickness range, a large value of the polarization is sustained. 

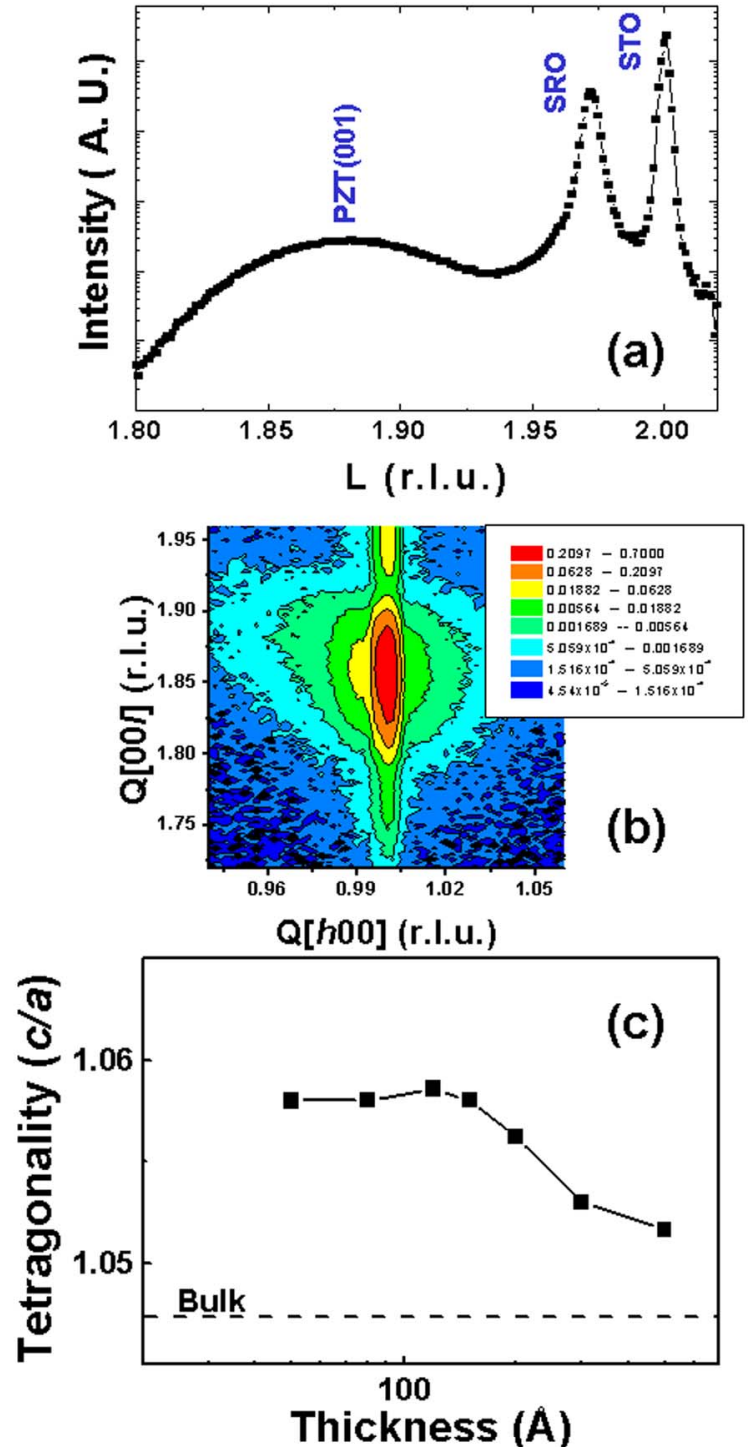

FIG. 2. (Color online) (a) Synchrotron x-ray (002) scan for the $50 \AA$ thick film. The peak position of (002) PZT, (200) SRO, and STO are labeled. (b) $h k l$ scan around (102) peak of the $50 \AA$ thick film. (c) Tetragonality measured as a function of film thickness. For films below $200 \AA$ thick a $1 \%$ increase in the tetragonality with respect to the bulk value is observed due to in-plane compression.

\section{B. Electrical measurements}

To ensure that the ultrathin films were of high quality we performed dc-AFM images to identify potential sources of leakage or breakdown. Here we show results from scans performed on the $50 \AA$ thick film. Figures 3(a)-3(d) are a set of typical $1 \times 1 \mu \mathrm{m}^{2}$ images (more than twice the area of the test capacitor devices) obtained for a $50 \AA$ PZT grown on SRO/STO. The legend on the right to each image shows the current passing through the sample. We see that up to $0.8 \mathrm{~V}$ $(\sim 1.6 \mathrm{MV} / \mathrm{cm})$ there is a uniform contrast indicating the lack of high conduction paths although the total current shows a slight increase. Only when the applied voltage is $1.5 \mathrm{~V}(3 \mathrm{MV} / \mathrm{cm})$ does the sample show breakdown, as indicated by the large number of white/light colored regions in the image. Therefore we concluded that even the thinnest films were able to withstand local electric fields almost as high as $3 \mathrm{MV} / \mathrm{cm}$.
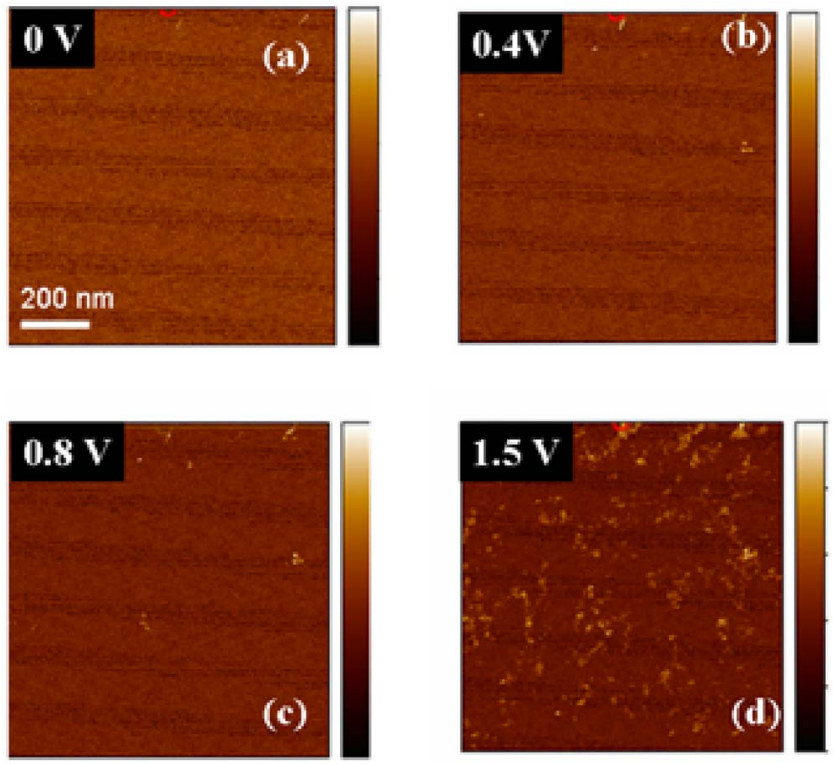

FIG. 3. (Color online) dc-AFM images at four different values of the bias voltage: (a) $0 \mathrm{~V}$, (b) $0.4 \mathrm{~V}$, (c) $0.8 \mathrm{~V}$, and (d) $1.5 \mathrm{~V}$. The images are a 1 $\times 1 \mu \mathrm{m}^{2}$ scan of the $50 \AA \mathrm{PZT} / \mathrm{SRO} / \mathrm{STO}$ sample. The color scale on the right of each image corresponds to ranges of (a) $0.00-0.549 \mathrm{nA}$, (b) $0.00-1.01 \mathrm{nA}$, (c) $0.00-2.4 \mathrm{nA}$, and (d) $0.00-8.54 \mathrm{nA}$, indicating the amount of current passing through the sample. The sample has low leakage, characterized by the absence of bright regions. Only after the application of high fields (larger than $2.4 \mathrm{MV} / \mathrm{cm}$ ) do conductive regions (white/light colored) appear, as seen in (d).

Figure 4(a) shows a set of switching transients recorded by the positive up negative down (PUND) sequence method measured at $2.5 \mathrm{MV} / \mathrm{cm}$ and $2 \mu \mathrm{s}$ pulse width for four typical thicknesses: $300,150,120$, and $80 \AA$. The inset to Fig. 4(a) shows the transients for the $50 \AA$ thick film. No resistance switching was observed for the ultrathin film, unlike reports on $\mathrm{PbZr}_{0.52} \mathrm{Ti}_{0.48} \mathrm{O}_{3}$ ultrathin films. ${ }^{24}$ Figure 4(a) shows a clear decrease in the magnitude as a function of decreasing thickness. Each switching transient was integrated to obtain the measured $\Delta P$. To ensure mathematical rigor we used the "double deflection" method. ${ }^{32}$ In Fig. 4(b) we show the results of the integration for each thickness. Films $150 \AA$ and thicker show a $\Delta P$ of $\sim 150 \mu \mathrm{C} / \mathrm{cm}^{2}$, almost twice the remnant bulk polarization. Below $150 \AA$, it progressively decreases to approximately $11 \mu \mathrm{C} / \mathrm{cm}^{2}$ for the $50 \AA$ film. Thus the polarization measurements reveal a systematic reduction in $\Delta P$ as a function of decreasing film thickness, i.e., a size effect as the thickness is scaled down. This again is very similar to the trend shown by BTO ultrathin films; very high tetragonality coupled with vanishing switched polarization. ${ }^{14}$ Further Fig. 4(c) plots the switched polarization as a function of applied field for each thickness. It is observed that films thicker than $150 \AA$ saturate to high switched polarization values; on the other hand the ultrathin films show no indications of saturation. Beyond these values of applied electric field significant breakdown was observed, hence convoluting the observed response. It is thus concluded that the ultrathin films show a highly pinned behavior and are unable to reach fully switched polarization even at extremely large electrical fields. 

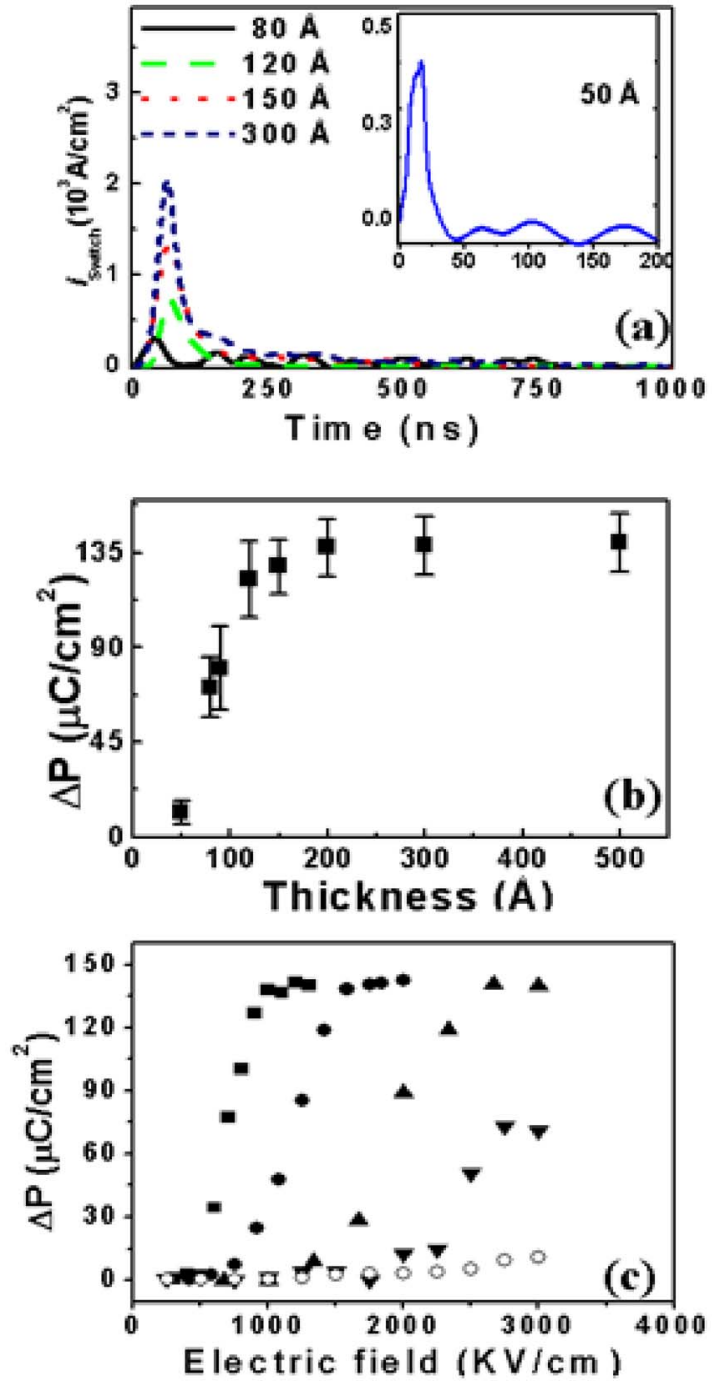

FIG. 4. (Color online) Switchable polarization as a function of film thickness. (a) Switching transients $(\Delta P)$ as a function of film thickness for a pulse width of $2 \mu \mathrm{s}$ and applied field of $2 \mathrm{MV} / \mathrm{cm}$. A clear decrease in the signal is observed as a function of decreasing thickness, thus suggesting a decrease in switchable polarization. The inset shows the switching transient for a $50 \AA$ thick film. (b) $\Delta P$ as a function of film thickness calculated by integration of the switching transients. As the film thickness decreases, the magnitude of switchable polarization decreases; thus the films, in contrast to the structural measurements, show a clear size effect in the polarization measurements. (c) Switched polarization as a function of applied field for

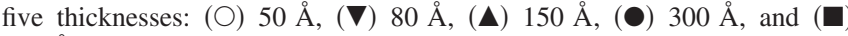
$500 \AA$. The thinner films show significantly high switching field and low switched polarization

In Fig. 5 the out-of-plane piezoelectric response $\left(d_{33}\right)$ is plotted as a function of thickness. The presence of a top electrode ensured a uniform distribution of the electric field. Figure 5(a) is a set of $d_{33}$ loops for five different thicknesses, from 40 to $300 \AA$. With decreasing film thickness, the piezoresponse decreases in a systematic and smooth manner. The piezoresponse is $60 \mathrm{pm} / \mathrm{V}$ for the $300 \AA$ film which drops to only $7 \mathrm{pm} / \mathrm{V}$ for the $40 \AA$ thick film. This thickness evolution is presented in Fig. 5(b). The error bars are standard deviations of the measurements. Comparing Fig. 4(b) and Fig. 5(b), a striking resemblance is seen for the $d_{33}$ thickness dependence vis-a-vis the polarization thickness dependence. This trend is not expected in the event of a monodomain
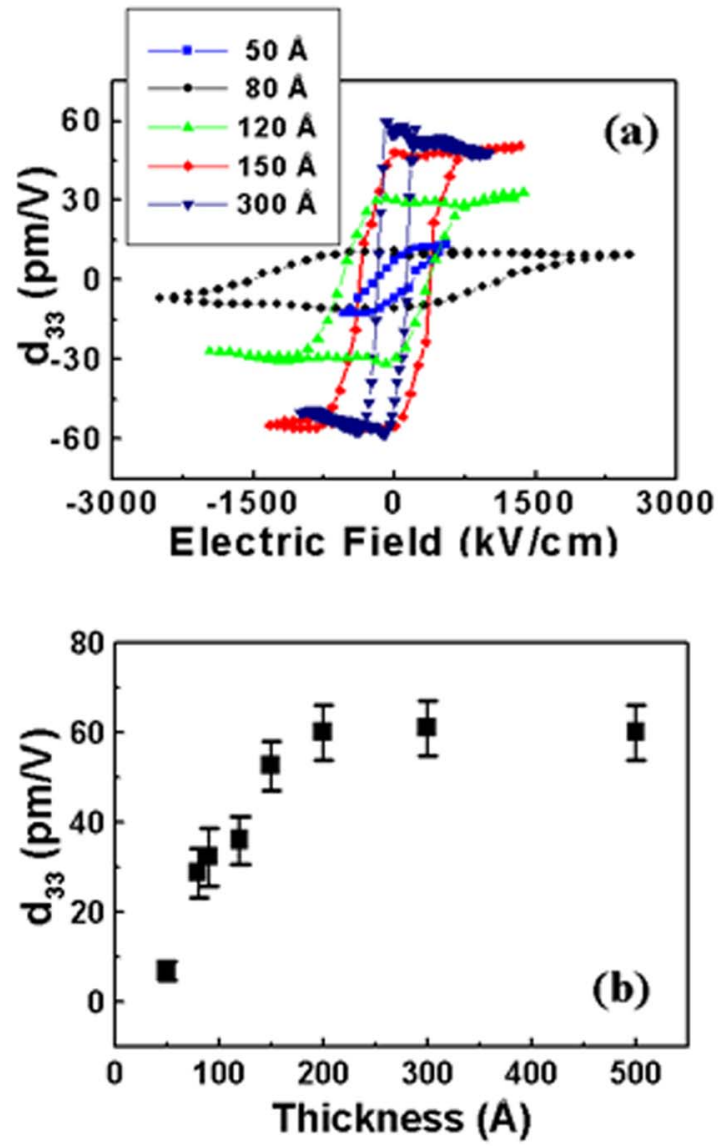

FIG. 5. (Color online) (a) Out-of-plane piezoelectric constant $d_{33}$ loops for five different thicknesses. It too shows a clear decrease with decreasing thickness although the coercive voltage increases. (b) $d_{33}$ as a function of film thickness. The trend shown by the $d_{33}$ is similar to the polarization: an unexpected decrease as a function of decreasing thickness. In the case of a real size effect, the $d_{33}$ is expected to increase with decreasing size.

ferroelectric to paraelectric real size driven phase transition; the piezoelectric response is expected to diverge with decreasing thickness in such a situation. ${ }^{38-40}$ Secondly, with decreasing thickness a systematic increase in the coercive field is observed, in compliance with previous reports. It increases from $150 \mathrm{kV} / \mathrm{cm}$ for the $300 \AA$ thick film to $1300 \mathrm{kV} / \mathrm{cm}$ for the $80 \AA \mathrm{film}$. The reason for this increase is still under debate; explanations range from crossover from nucleation and growth of domains to intrinsic switching ${ }^{6}$ to the presence of a strong depolarizing field which must be corrected for. ${ }^{22}$ Also with decreasing thickness the electric field dependence of the $d_{33}$ progressively decreases while the tilt of the loop increases. The reason for this increasing tilt is attributed to the presence of passive layers as demonstrated by Tagantsev et al. in a series of papers. ${ }^{41,42}$ The analysis of the tilting of the loop forms a more detailed study in itself and will be discussed elsewhere. ${ }^{43}$

Therefore, the most important features of the above experimental observations can be summarized as follows: (i) an enhancement of tetragonality of about $1 \%$ with respect to the bulk for films thinner than $200 \AA$, (ii) a lack of scaling for tetragonality with thickness in the sub-100- $\AA$ range, (iii) a clear decrease in the magnitude of the switchable polarization with decreasing thickness, and (iv) a substantial reduc- 
tion of the out-of-plane piezoelectric response for the thinnest films. Due to the well-known strong polarization-strain coupling in ferroelectric perovskite oxides, explicitly checked by first-principles calculations at the bulk level for $\mathrm{PZT}^{44}$ (i) and (ii) suggest the existence of a ferroelectric polarization in the system, whereas (iii) and (iv) point to reduced ferroelectricity tending towards a paraelectric ground state with no net polarization. Thus, both the polarization and piezoelectric properties are in direct contradiction to our structural data, which show negligible size effects in the tetragonality.

\section{Theory}

To resolve this apparent inconsistency, we have carried out first-principles effective Hamiltonian simulations. Within this approach, the energy is written as a low-order Taylor expansion of the bulk energy around the paraelectric cubic phase in terms of selected degrees of freedom. Our starting point was the model Hamiltonian developed by Waghmare and Rabe for bulk PTO, ${ }^{45}$ where the expansion is made in terms of the ionic displacements associated to the lattice Wannier function of the soft phonon branch, and the macroscopic strain. The parameters of the expansion are obtained by fitting first-principles results. We use the parameters for pure PTO (Ref. 45) as the main effect of the $20 \% \mathrm{Zr}$ alloying is to only slightly shift the transition temperature and spontaneous polarization. Then, the model Hamiltonian was extended to deal with thin films following a well-established precedent, ${ }^{18,23}$ where the structure and properties of the thin film are obtained from simulation of the bulk material with the corresponding epitaxial strain constraint (in the present case, the in-plane lattice vectors are fixed to match the cubic STO substrate, $3.905 \AA$ ), and macroscopic electric field resulting from the incomplete screening by real metallic electrodes. The details of the generalization of the model Hamiltonian to thin films are given in Ref. 23. Previous firstprinciples calculations ${ }^{18}$ have shown that short-circuit boundary conditions across the entire system result in a nonzero macroscopic field in the thin film that depends linearly on the polarization of the thin film and is inversely proportional to the thickness of the ferroelectric layer. This residual depolarization field $\mathcal{E}_{d}$ couples with the polarization, yielding an extra electrostatic energy that tends to suppress the polarization. ${ }^{20}$ Only the net component of the out-of-plane polarization is considered here to compute the homogeneous depolarization field $\mathcal{E}_{d}$ and the corresponding contributions to the energy. ${ }^{46}$

The thickness dependence of the normal average polarization $P$, tetragonality $c / a$, and the out-of-plane piezoelectric constant $\left(d_{33}\right)$ at room temperature, obtained by classical Monte Carlo simulations for a $12 \times 12 \times 12$ supercell, are presented in Fig. 6. We have performed simulations for larger supercells and confirmed that the results are independent of cell size. A sharp monodomain-polydomain phase transition, driven by $\mathcal{E}_{d}$, is detected at a thickness around $150 \AA$. This is similar to phase transitions reported in Refs. 21 and 47-50. The phase transition results from the competition between the compressive strain imposed by the sub-
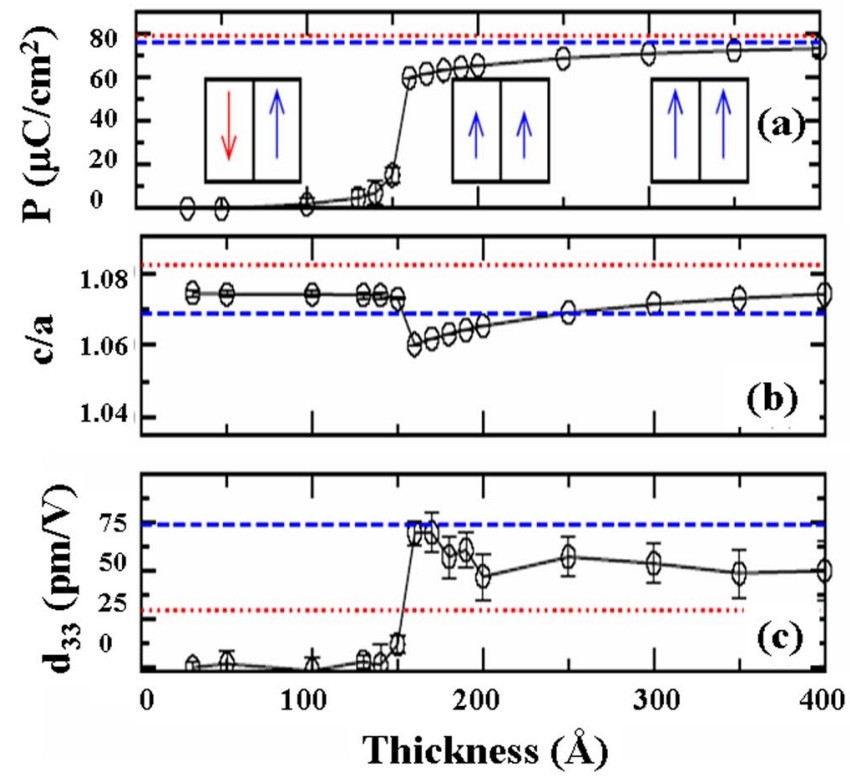

FIG. 6. (Color online) Thickness dependence of the normal average polarization $P$, the tetragonality $c / a$, and the out-of-plane piezoelectric constant $d_{33}$ at room temperature. Values of these quantities at the bulk level are indicated by the dashed lines for the unstrained configuration and by the dotted lines for a geometry under the strain imposed by the substrate. The evolution of the domain structure, from a monodomain configuration at large thicknesses, where the depolarization field $\mathcal{E}_{d}$ is small, to a $180^{\circ}$ stripe domains to minimize the energy associated with $\mathcal{E}_{d}$ is presented in the inset. Standard deviation distributions, obtained from averaging over different numbers of Monte Carlo steps in many simulations, starting with different seeds for the random number generator, are indicated by error bars.

strate, which tends to stabilize the tetragonal phase ${ }^{51-53}$ and the out-of-plane polarization, ${ }^{52}$ and a depolarization field that tends to suppress the latter. As the film gets thinner, the residual field in the ferroelectric grows and, consequently, both the polarization ${ }^{18}$ and the tetragonality ${ }^{23}$ are progressively reduced [see Figs. 6(a) and 6(b)]. Below the critical thickness the system breaks up into equal-width $180^{\circ}$ stripe domains (net polarization zero) to minimize the energy associated with $\mathcal{E}_{d}$ [Figs. 7(a)-7(c)]. This domain structure has been observed and characterized in ultrathin PTO films grown on insulating substrates. ${ }^{9,11}$ Here, we propose its existence as the mechanism for compensation of the depolarization field for ferroelectric layers sandwiched between metallic electrodes as well. Though the net switchable polarization is zero, each domain exhibits the bulk strained polarization and tetragonality $c / a, 1.25 \%$ larger than in the unstrained sample, explaining the apparent inconsistency of the experimental data. Moreover, since the out-of-plane piezoelectric response is also directly proportional to the polarization inside each domain, its average also tends to cancel for films thinner than the threshold thickness, explaining the drop of $d_{33}$ experimentally observed [Fig. 6(c)].

One might expect that a transition from the $180^{\circ}$ domain state to the uniformly polarized state could be induced by the application of a sufficiently high electric field; however, experimentally this is not observed as shown by Fig. 4(c). Simulations of the response of the system under high electric field were carried out to check the strong nature of the pinned domain state. In Fig. 8 we present the results of the 

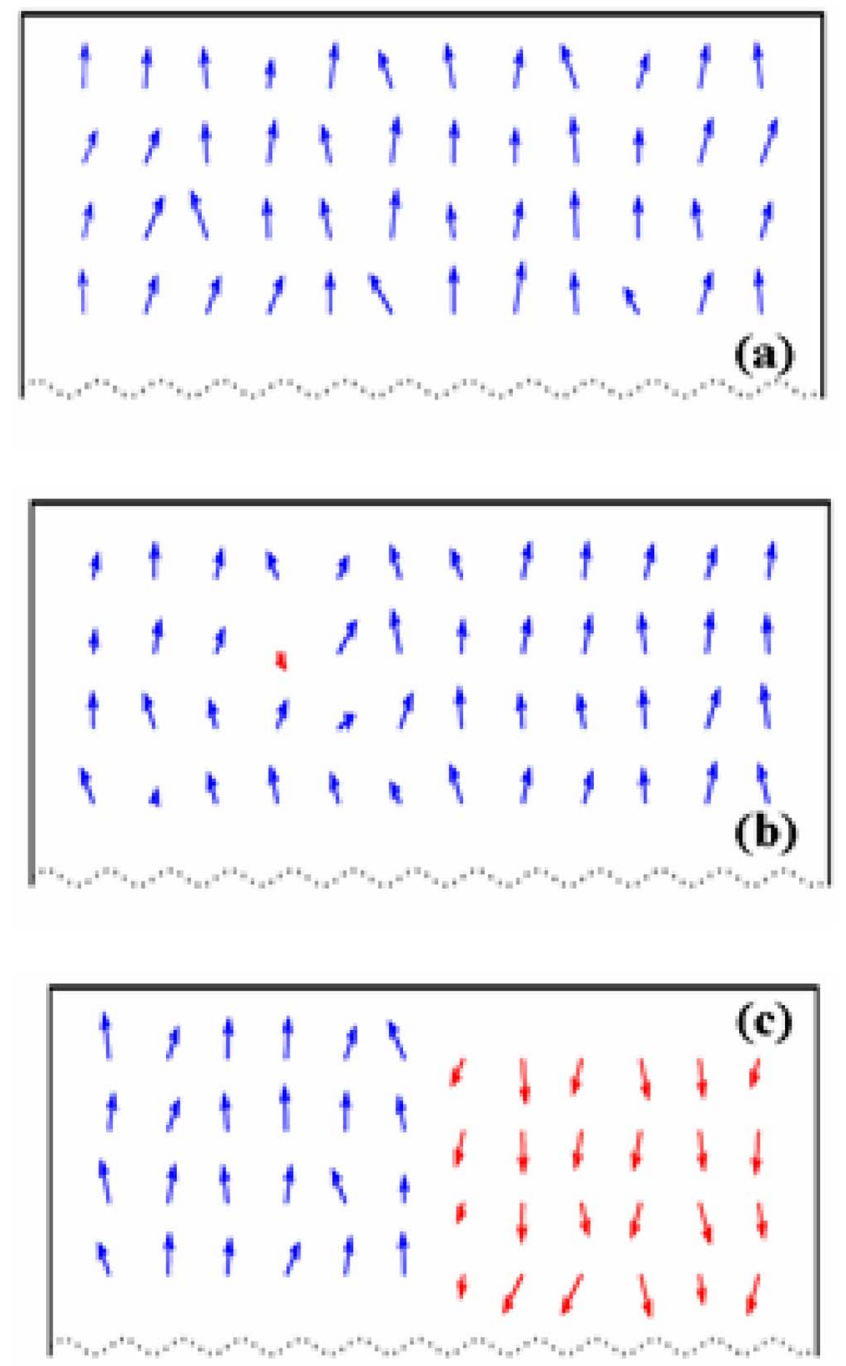

FIG. 7. (Color online) Local mode displacements of the cells situated in a transverse cut of the film at different thicknesses: (a) $350 \AA$, (b) $150 \AA$, and (c) $60 \AA$. The arrows give the direction of the displacement projected on a $y z$ plane, where $z$ is the normal direction to the interface, and the arrows' length indicates the projected magnitudes. A sharp monodomainpolydomain phase transition is observed at a thickness around $150 \AA$ A. Above this critical thickness (panel a), the local mode is reduced, and even locally reversed by the effect of the residual depolarization field. Below the critical thickness, domains of opposite polarization nucleate and grow with decreasing thickness till a 50/50 configuration, with no net polarization, is formed.

Monte Carlo simulations under the presence of a field of $3 \mathrm{MV} / \mathrm{cm}$. It is seen that even under such high fields, the theoretically calculated switched polarization for the sub$100-\AA ̊$ thick films is very small. In order to explain this behavior, it is necessary to understand the different energies involved. Let us start with a 50/50 domain structure as the one described in the preceding paragraph. In such a configuration, the switchable polarization is zero. The net polarization at the surface $\langle P\rangle$ also vanishes, and consequently there is no depolarization field $\mathcal{E}_{d}$, according to the expression for the depolarizing field given in Ref. 23.

$$
\langle P\rangle=0,
$$

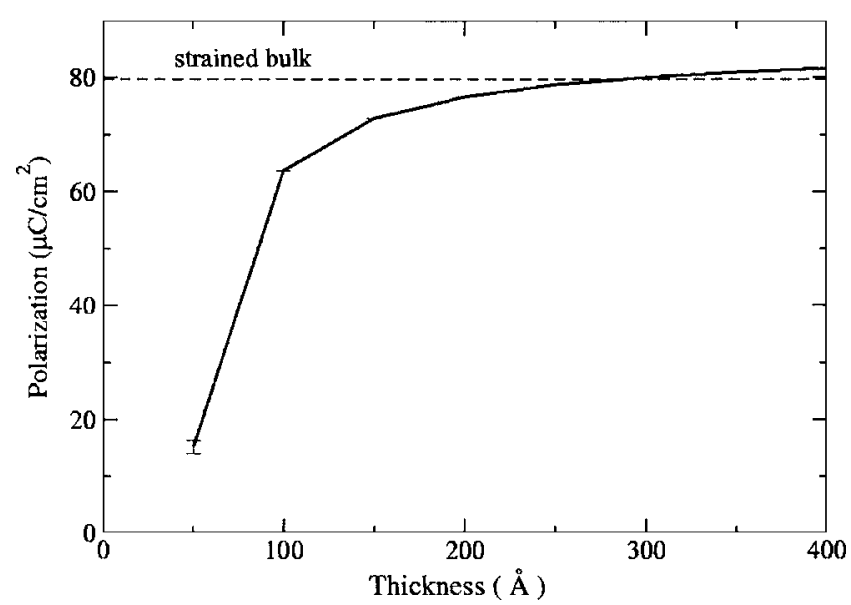

FIG. 8. Thickness dependence of the normal average polarization under the effect of an external applied field of $3 \mathrm{MV} / \mathrm{cm}$ at room temperature. Even at such high fields, the $180^{\circ}$ domains remain pinned, thus resulting in a reduced average polarization. The bulk strained value in zero field (dashed line) is presented for comparison. The error bars have the same meaning as in Fig. 6.

$$
\mathcal{E}_{d}=\frac{-2 \lambda\langle P\rangle}{\varepsilon_{0} d}=0
$$

where $\lambda$ stands for the effective screening length of the electrode and $d$ is the thickness of the ferroelectric thin film (we use SI units).

Now, let us switch on an external field $\mathcal{E}_{\text {appl }}$ pointing up. This field couples with the polarization inside each domain, and the corresponding electrostatic energy coupling $E_{\text {appl }}$ will be given by

$$
\begin{aligned}
E_{\text {appl }} & =-\int_{\Omega} \mathcal{E}_{\text {appl }} P d \Omega \\
& =-\int_{\Omega_{\text {up }}} \mathcal{E}_{\text {appl }} P d \Omega_{\text {up }}-\int_{\Omega_{\text {down }}} \mathcal{E}_{\text {appl }} P d \Omega_{\text {down }} \\
& =-\mathcal{E}_{\text {appl }} P\left(\Omega_{\text {up }}-\Omega_{\text {down }}\right) \\
& =-\mathcal{E}_{\text {appl }}\langle P\rangle \Omega
\end{aligned}
$$

where $\Omega_{\text {up }}$ and $\Omega_{\text {down }}$ stand for the volume of the domains up and down, respectively, $\Omega$ refers to the whole volume of the sample, and we have supposed that both the local polarization (the same in magnitude inside each domain) and the applied field are homogeneous. In the previous expression, we have made use of the fact that $\mathcal{E}_{\text {appl }}$ is parallel to the polarization up and antiparallel to the polarization down. Clearly, the electrostatic energy is linear in the net polarization, the domain up being energetically favorable against domain down. That means that in the absence of a depolarization field, the system will minimize the energy by flipping all the domains up completely.

However, as soon as the domain wall is displaced, a net polarization $\langle P\rangle$ appears, giving rise to a proportional depolarization field $\mathcal{E}_{d}$, as it is shown in Eq. (1). The corresponding energy coupling $E_{\text {dep }}$ is positive in sign (that is, it tends to suppress the polarization) and quadratic on the net polarization, ${ }^{23,39}$ 

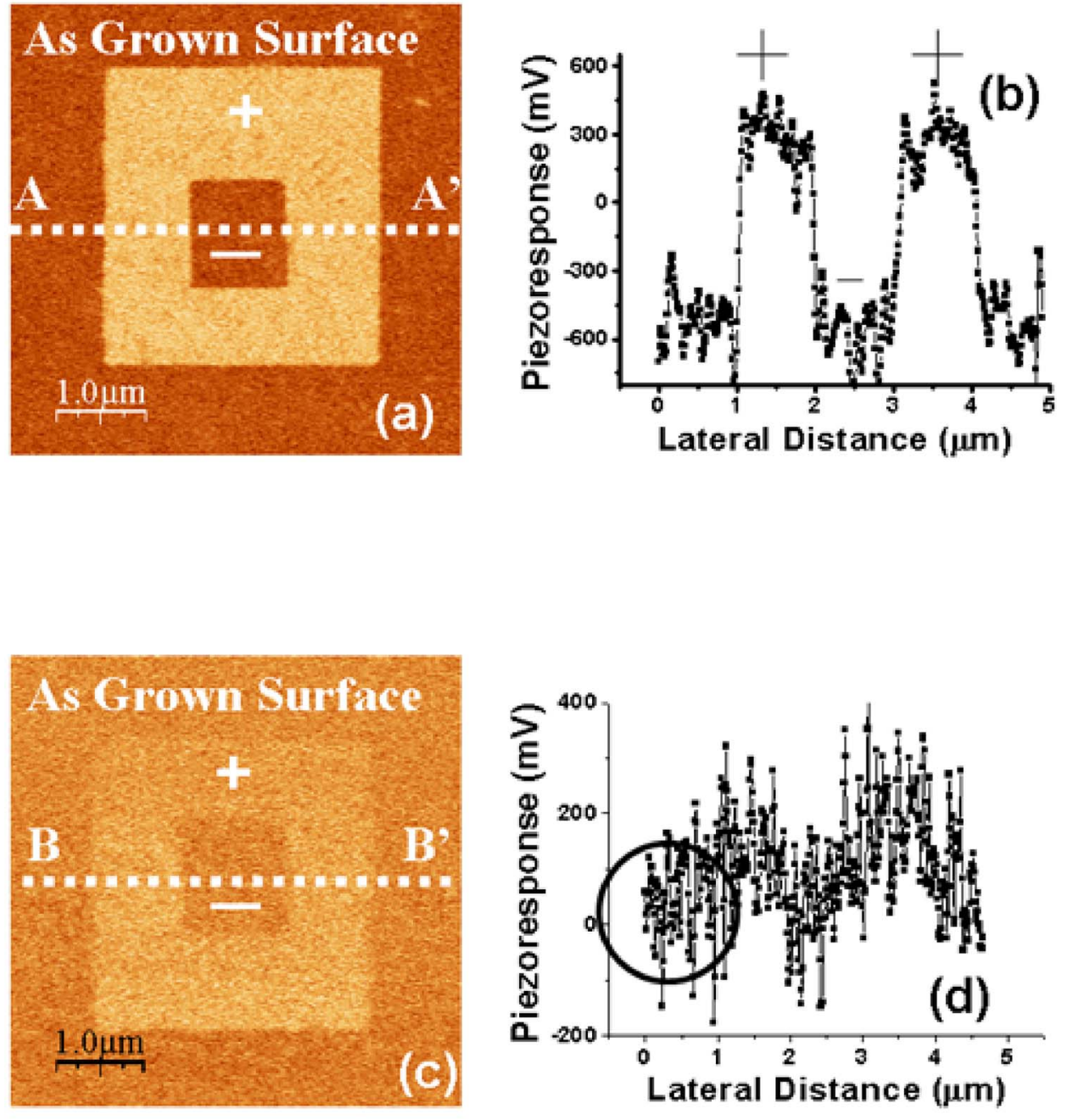

$$
E_{\mathrm{dep}}=+\frac{2 \lambda\langle P\rangle^{2}}{\varepsilon_{0} d} .
$$

necessitates application of an electric field) and not ferroelectric hysteresis loops. Indeed when the simulations are performed under an applied electric field, the drop is gradual as observed in Fig. 8.

Secondly, the experimentally observed remnant piezoelectric response for the sub-100- $\AA$ films (although only $7-15 \mathrm{pm} / \mathrm{V}$ ) is not zero, whereas the theoretical simulations predict a vanishing piezoresponse on account of the polydomain state. This is because the remnant state in the loop is reached only after sweeping to the maximum positive electric field, on its way to maximum negative electric field. Thus, there is a history dependent path involved, which cannot be accounted for by the theoretical simulations.

To check the presence of a $180^{\circ}$ polydomain state we performed PFM imaging of the as grown samples. We performed synchrotron experiments as well to check the presence of satellite peaks as demonstrated by Fong and co-workers. ${ }^{9,11}$ However, the Pohang beam line does not have adequate beam energy to resolve the peaks. Therefore we retorted to the technique of PFM. Figure 9 compares the PFM images of two thicknesses, 150 and $50 \AA$ A. Figure 9(a) is a $5 \times 5 \mu \mathrm{m}^{2} \mathrm{PFM}$ scan of the $150 \AA$ film. At the center a $3 \times 3 \mu \mathrm{m}^{2}$ region is switched by application of $+5 \mathrm{~V}$ and within that a $1 \times 1 \mu \mathrm{m}^{2}$ region is switched by applying a reverse bias of $-5 \mathrm{~V}$. Time dependent scans of the images 
did not reveal any decrease or relaxation of the piezoresponse, suggesting that the written regions were extremely stable. ${ }^{7}$ Figure 9 (b) is the profile along section $A A^{\prime}$ as drawn in Fig. 9(a). The following interesting observations can be made.

(a) Outside the written zone line scan of the virgin region is uniformly negative, which suggests that the as grown film has a uniform polarity. In other words the $150 \AA$ thick film is monodomain. This is in good agreement with the theoretical predictions.

(b) Application of the positive bias (region marked with "+") creates regions of opposite contrasts and changes the sign of the piezoresponse signal [as seen in Fig. 9(b)] to positive but the magnitude is approximately the same, demonstrating that regions can be fully switched. This is in good agreement with the quantitative piezoelectric and the polarization measurements discussed earlier.

(c) Application of negative bias (region marked as "-") reverts the piezoresponse and hence the polarization back to the original contrast.

Figure 9(c) is the PFM scan for the $50 \AA$ thick film with identically repeated write experiments. Figure $9(\mathrm{~d})$ plots the profile for line $B B^{\prime}$ in Fig. 9(c). It is found that in comparison to the $150 \AA$ sample images, the result for the $50 \AA$ film is significantly different in the following manner.

(a) Most importantly the virgin surface shows a piezoresponse very close to zero. In Fig. 9(d) this is the region inside the circle. This indicates that the film has a polydomain state such that the response is approximately zero.

(b) On application of the positive bias, there is a piezoresponse signal and the magnitude is positive. This indicates that the film can be switched; however, the magnitude of the written region is less than $20 \%$ of the $150 \AA$ region, in agreement with the trend shown by quantitative PFM.

(c) On applying the reverse negative bias, it is observed that central region $(-)$ does not switch back to a negative state but, in fact, hovers around zero, i.e., returns to the virgin state. This implies that the pinned polydomain state is stable favored to a uniformly negative monodomain state. This is slightly different from our quantitative results, where a piezoresponse is observed, similar in magnitude to the positive cycle. This difference may arise from the asymmetry of electrodes used in imaging experiments.

We thus conclude that the PFM studies indicate that the sub-100- $\AA$ ultrathin film is polydomain, thereby validating the conclusions of our theoretical simulations.

It could be surmised that the presence of ionic defects (e.g., oxygen vacancies) or electronic charge carriers could possibly lead to elongated lattice parameters ${ }^{54}$ or would pin domain walls ${ }^{55,56}$ and therefore reduce the measured switchable polarization. While we cannot fully neglect the effect of oxygen vacancies, generally such ionic vacancies are ex- pected to increase leakage currents dramatically. ${ }^{57}$ This is not the case as shown by the dc-AFM images. Additionally x-ray photoemission spectroscopy (XPS) measurements did not show any measurable changes in the $\mathrm{Pb}^{+2}$ or $\mathrm{Ti}^{+4}$ peak positions as a function of film thickness; therefore we also precluded changes in the electronic states of the cations as the major cause for the reduction in polarization.

\section{CONCLUSIONS}

In summary, we show in ultrathin SRO/PZT/SRO heterostructures a strikingly anomalous behavior: virtually no scaling of the tetragonality down to $50 \AA$ thickness accompanied by a systematic reduction of the switchable polarization and the out-of-plane piezoelectric constant. Firstprinciples simulations predict the formation of a $180^{\circ}$ polydomain state for the thinnest films due to the presence of a residual depolarization field. The presence of a polydomain state is experimentally indicated by PFM images. Both the simulations and experiments suggest that this polydomain state is pinned and therefore explain the observed anomaly. Our studies on epitaxial perovskite ferroelectric thin films therefore indicate that although ferroelectricity is maintained down to nanometer level thickness, switchable polarization is severely affected.

It is also pertinent here to ask why polarization saturation cannot be observed in our films where as no size effects and complete saturation were reported for ultrathin polyvinylidene difluoride (PVDF) polymer films. ${ }^{6}$ The reason for this may perhaps be that the nature of ferroelectricity in PVDF is different compared to PZT; this is a topic of our current investigation. Furthermore why resistance switching is not observed for this composition while it is seen for other PZT compositions needs further investigations.

\section{ACKNOWLEDGMENTS}

This work was supported by NSF MRSEC Grant No. DMR-00-80008 and a NSF-DFG collaborative Grant No. DMR-02-44288. The work at Liège and Juelich was supported by the VolkswagenStiftung (1/77 737). One of the authors (Ph.G.) also acknowledges the support of FNRSBelgium (Contract No. 2.4562.03) and the European Network of Excellence "FAME" (Contract No. FP6-500156-1). Another author (V.N.) acknowledges the Alexander von Humboldt foundation for his stay in FZ-Juelich, Germany. The authors acknowledge helpful discussions with Dr. H. H. Kohlstedt and Dr. M. Dawber and the help of Professor Szot for the dc-AFM images.

${ }^{1}$ J. F. Scott and C. A. P. de Araujo, Science 246, 1400 (1989).

${ }^{2} \mathrm{R}$. Waser, Nanoelectronics and Information Technology (Wiley-VCH, Weinheim, 2003).

${ }^{3}$ T. M. Shaw, S.-T. McKinstry, and P. C. McIntyre, Annu. Rev. Mater. Sci. 30, 263 (2000).

${ }^{4}$ C. H. Ahn, K. M. Rabe, and J.-M. Triscone, Science 303, 488 (2004).

${ }^{5} \mathrm{Ph}$. Ghosez and J. Junquera, in Handbook for Theoretical and Computational Nanotechnology, edited by M. Reith and W. Schommers (American Scientific Publishers, Oswego, NY, 2006).

${ }^{6}$ A. V. Bune, V. M. Fridkin, S. Ducharme, L. M. Blinov, S. P. Palto, A. V. Sorokin, S. G. Yudin, and A. Zlatkin, Nature (London) 391, 874 (1998). ${ }^{7}$ Th. Tybell, C. H. Ahn, and J.-M. Triscone, Appl. Phys. Lett. 75, 856 (1999). 
${ }^{8}$ N. Yanase, K. Abe, N. Fukushima, and T. Kawakubo, Jpn. J. Appl. Phys., Part 1 38, 5305 (1999).

${ }^{9}$ S. K. Streiffer et al., Phys. Rev. Lett. 89, 067601 (2002).

${ }^{10}$ M.-W. Chu, I. Szafraniak, R. Scholz, C. Harnagea, D. Hesse, M. Alexe, and U. Gösele, Nat. Mater. 3, 87 (2004).

${ }^{11}$ D. D. Fong, G. B. Stephenson, S. K. Streiffer, J. A. Eastman, O. Auciello, P. H. Fuoss, and C. Thompson, Science 304, 1651 (2004).

${ }^{12}$ H. Ishiwara, M. Okuyama, and Y. Arimoto, Ferroelectric Random Access Memories: Fundamentals and Applications (Springer-Verlag, 2004), Vol. 93.

${ }^{13}$ V. Nagarajan et al., Appl. Phys. Lett. 84, 5225 (2004).

${ }^{14}$ Y. S. Kim et al., Appl. Phys. Lett. 86, 102907 (2005).

${ }^{15}$ R. Kretschmer and K. Binder, Phys. Rev. B 20, 1065 (1979).

${ }^{16} \mathrm{Ph}$. Ghosez and K. M. Rabe, Appl. Phys. Lett. 76, 2767 (2000).

${ }^{17}$ A. G. Zembilgotov, N. A. Pertsev, H. Kohlstedt, and R. Waser, J. Appl. Phys. 91, 2247 (2002).

${ }^{18}$ J. Junquera and $\mathrm{Ph}$. Ghosez, Nature (London) 422, 506 (2003).

${ }^{19}$ M. D. Glinchuk, E. A. Eliseev, and V. A. Stephanovich, Physica B 322, 356 (2002).

${ }^{20}$ I. P. Batra, P. Wurfel, and B. D. Silverman, J. Vac. Sci. Technol. 10, 687 (1973).

${ }^{21}$ Z. Q. Wu, N. D. Huang, Z. R. Liu, J. Wu, W. H. Duan, B. L. Gu, and X. W. Zhang, Phys. Rev. B 70, 104108 (2004).

${ }^{22}$ M. Dawber, P. Chandra, P. B. Littlewood, and J. F. Scott, J. Phys.: Condens. Matter 15, L393 (2003).

${ }^{23}$ C. Lichtensteiger, J.-M. Triscone, J. Junquera, and Ph. Ghosez, Phys. Rev. Lett. 94, 047603 (2005).

${ }^{24}$ J. R. Contreras, H. Kohlstedt, U. Poppe, C. Buchal, N. A. Pertsev, and R. Waser, Appl. Phys. Lett. 83, 495 (2003).

${ }^{25}$ H. Qu, W. Yao, T. Garcia, J. Zhang, A. V. Sorokin, S. Ducharme, P. A. Dowben, and V. M. Fridkin, Appl. Phys. Lett. 82, 4322 (2003).

${ }^{26}$ M. Y. Zhuravlev, R. F. Sabirianov, S. S. Jaswal, and E. Y. Tsymbal, Phys. Rev. Lett. 94, 246802 (2005).

${ }^{27}$ K. M. Indlekofer and H. Kohlstedt, Europhys. Lett. 72, 282 (2005).

${ }^{28}$ B. S. Simpkins, E. T. Yu, P. Waltereit, and J. S. Speck, J. Appl. Phys. 94 1448 (2003).

${ }^{29}$ M. Porti, M. Nafria, X. Aymerich, A. Olbrich, and B. Ebersberger, Microelectron. Eng. 9, 265 (2001)

${ }^{30}$ D. M. Schaadt, E. T. Yu, V. Vaithyanathan, and D. G. Schlom, J. Vac. Sci. Technol. B 22, 2030 (2004).

${ }^{31}$ S. V. Kalinin and D. A. Bonnell, Nano Lett. 4, 555 (2004)

${ }^{32}$ S. Prasertchoung, V. Nagarajan, Z. Ma, R. Ramesh, J. S. Cross, and M. Tsukada, Appl. Phys. Lett. 84, 3130 (2004).

${ }^{33}$ J. A. Christman, J. R. R. Woolcott, A. I. Kingon, and R. J. Nemanich,
Appl. Phys. Lett. 73, 3851 (1998).

${ }^{34}$ V. Nagarajan et al., Nat. Mater. 2, 43 (2003)

${ }^{35}$ A. T. J. van Helvoort, O. Dahl, B. G. Soleim, R. Holmestad, and Th. Tybell, Appl. Phys. Lett. 86, 092907 (2005).

${ }^{36}$ S. Stemmer, S. K. Streiffer, F. Ernst, and M. Ruhle, Philos. Mag. A 71, 713 (1995).

${ }^{37}$ R. E. Cohen, Nature (London) 358, 136 (1992).

${ }^{38}$ J. Junquera (unpublished).

${ }^{39}$ M. E. Lines and A. M. Glass, Principles and Applications of Ferroelectrics and Related Materials (Oxford University Press, Oxford, 1977).

${ }^{40}$ W. L. Zhong, B. D. Qu, P. L. Zhang, and Y. G. Wang, Phys. Rev. B 50, 12375 (1994).

${ }^{41}$ A. K. Tagantsev, V. O. Sherman, K. F. Astafiev, J. Venkatech, and N. Setter, J. Electroceram. 11, 5 (2003).

${ }^{42}$ A. K. Tagantsev, M. Landivar, E. Colla, and N. Setter, J. Appl. Phys. 78, 2623 (1995).

${ }^{43}$ S. Zhong, S. P. Alpay, and V. Nagarajan, Mater. Res. 21, 1600 (2006).

${ }^{44}$ First-principles density-functional calculations on bulk $\mathrm{Pb}\left(\mathrm{Zr}_{0.2} \mathrm{Ti}_{0.8}\right) \mathrm{O}_{3}$ confirm that the polarization along the $c$ axis steadily grows with the increasing of the tetragonal order parameter over a wide range of $c / a$; $\mathrm{U}$. V. Waghmare (private communication).

${ }^{45}$ U. V. Waghmare and K. M. Rabe, Phys. Rev. B 55, 6161 (1997).

${ }^{46}$ We point out that the absence of the stray energy spoils the applicability of our model to thickness below the domain width (Refs. 47 and 48) (in our simulations half of a supercell, that is, around $24 \AA$ ). A more detailed model is needed for the thinnest films.

${ }^{47}$ A. M. Bratkovsky and A. P. Levanyuk, Phys. Rev. Lett. 84, 3177 (2000).

${ }^{48}$ A. M. Bratkovsky and A. P. Levanyuk, Phys. Rev. B 63, 132103 (2001).

${ }^{49}$ M. G. Stachiotti, Appl. Phys. Lett. 84, 251 (2004).

${ }^{50}$ I. Kornev, H. Fu, and L. Bellaiche, Phys. Rev. Lett. 93, 196104 (2004).

${ }^{51}$ N. A. Pertsev, A. G. Zembilgotov, and A. K. Tagantsev, Phys. Rev. Lett. 80, 1988 (1998).

${ }^{52}$ K. J. Choi et al., Science 306, 1005 (2004).

${ }^{53}$ O. Diéguez, S. Tinte, A. Antons, C. Bungaro, J. B. Neaton, K. M. Rabe, and D. Vanderbilt, Phys. Rev. B 69, 212101 (2004).

${ }^{54}$ W. L. Warren, G. E. Pike, K. Vanheusden, D. Dimos, B. A. Tuttle, and J. Robertson, J. Appl. Phys. 79, 9250 (1996).

${ }^{55}$ W. L. Warren, D. Dimos, B. A. Tuttle, G. E. Pike, R. W. Schwartz, P. J. Clews, and D. C. McIntyre, J. Appl. Phys. 77, 6695 (1995).

${ }^{56}$ W. L. Warren, D. Dimos, B. A. Tuttle, R. D. Nasby, and G. E. Pike, Appl. Phys. Lett. 65, 1018 (1994).

${ }^{57}$ G. Catalan, M. H. Corbett, R. M. Bowman, and J. M. Gregg, Appl. Phys. Lett. 74, 3035 (1999) 